OPEN ACCESS

Edited by:

Nicholas Pavlidis,

University of loannina, Greece

Reviewed by:

Ghamartaj Hossein,

University of Tehran, Iran

Antonella Tomassetti,

Fondazione Istituto nazionale Tumor

Milan (IRCCS), Italy

*Correspondence:

Tianijao Lyu

Itj12196@rih.com.cn

Weiwei Feng

fww12066@rih.com.cn

${ }^{t}$ These authors have contributed equally to this work and share

first authorship

Specialty section: This article was submitted to

Gynecological Oncology,

a section of the journal

Frontiers in Oncology

Received: 03 April 2021 Accepted: 31 August 2021

Published: 21 September 2021

Citation:

Jiang $Y$, Zhou T, Shi Y, Feng $W$ and Lyu T (2021) A SMYD3/ITGB6/

TGF 1 1 Positive Feedback Loop

Promotes the Invasion and Adhesion

of Ovarian Cancer Spheroids.

Front. Oncol. 11:690618.

doi: 10.3389/fonc.2021.690618

\section{A SMYD3/ITGB6/TGF $\beta 1$ Positive Feedback Loop Promotes the Invasion and Adhesion of Ovarian Cancer Spheroids}

\author{
Yahui Jiang ${ }^{\dagger}$, Tianyu Zhou ${ }^{\dagger}$, Yiwen Shi, Weiwei Feng ${ }^{*}$ and Tianjiao Lyu * \\ Department of Gynecology and Obstetrics, Ruijin Hospital, School of Medicine, Shanghai Jiaotong University, Shanghai, China
}

Background: Implantation metastasis is the main means of dissemination in ovarian cancer. Our previous studies showed that SET and MYND domain-containing protein 3 (SMYD3) expression was higher in ovarian cancer spheroids than in monolayers. SMYD3 enhancement of spheroid invasion and adhesion is mediated by the downstream effectors ITGB6 and ITGAM. However, the potential mechanisms underlying the SMYD3/integrinmediated invasion and adhesion of spheroids still need to be explored.

Methods: Western blotting was used to examine the expression of SMYD3, ITGB6 and downstream molecules under different treatments. Immunofluorescence was used to detect the expression of F-actin, E-cadherin and N-cadherin. Anti-ITGB6 antibody-based inhibition and dual-luciferase reporter assays were used to confirm the binding between ITGB6 and latent TGF $\beta 1$. Transwell invasion, adherence and 3D tumor spheroid invasion assays were employed to test the effects of TGF $\beta 1$ on the invasion and adhesion of ovarian cancer spheroids. ELISA was performed to assess the release of latent TGF $\beta 1$ from ovarian cancer spheroids.

Results: SMYD3 and ITGB6 activated the TGF $\beta 1 /$ Smad3 pathway and then induced the upregulation of Snail, Vimentin and $\mathrm{N}$-cadherin and the downregulation of E-cadherin in 3D-cultured ovarian cancer spheroids. In this process, latent TGF $\beta 1$ could bind to ITGB6 and become activated to stimulate the Smad3 pathway. Moreover, SMYD3 and ITGB6 could facilitate the release of latent TGF $\beta 1$ from 3D-cultured ovarian cancer spheroids. Interestingly, TGF $\beta 1$ could promote the expression of SMYD3 and ITGB6 via feedback. This positive feedback loop could further amplify the biological effect and promote the invasion and adhesion of ovarian cancer spheroids.

Conclusion: Our results demonstrated that the SMYD3/ITGB6/TGF $\beta 1$-Smad3 positive feedback loop could promote the invasion and adhesion of ovarian cancer spheroids by upregulating the expression of $\mathrm{N}$-cadherin, Snail, and Vimentin and downregulating the expression of E-cadherin. Thus, our study unmasked the mechanism of SMYD3- and ITGB6-induced ovarian cancer metastasis and provides new ideas for targeted ovarian cancer treatment.

Keywords: ovarian cancer spheroids, metastasis, SMYD3, ITGB6, TGFß1 


\section{INTRODUCTION}

Ovarian cancer is the most lethal gynecological malignancy worldwide, and the lifetime risks of developing and dying from ovarian cancer are $0.72 \%$ and $0.45 \%$, respectively $(1,2)$. Characteristically, over $70 \%$ of epithelial ovarian cancer (EOC) patients have intra-abdominal dissemination involving local invasion of pelvic and abdominal organs but rarely have vascular metastasis at diagnosis (3). This is mainly because of the unique characteristics of implantation metastasis in EOC, including detachment from the original lesion, formation of spheroids in ascites, adherence to peritoneal mesothelial cells, and anchoring to the stroma. In addition, biological features such as the adhesion and invasion potential of spheroid cells also account for EOC recurrence (4). Therefore, more research has cast light on the mechanism of EOC implantation metastasis in recent years. The phenotype and gene expression profile of EOC cells constantly change during the processes of shedding, suspension, re-adhesion and proliferation to adapt to the microenvironment.

SET and MYND domain-containing protein 3 (SMYD3) is a histone methyltransferase that acts as a gene transcriptional regulator, participating in the methylation of various histone and nonhistone targets (5). Aberrant SMYD3 expression is found during carcinogenesis in multiple cancers, such as prostate cancer, breast cancer and colorectal cancer, suggesting its essential roles in tumor initiation and progression (6).

In our previous studies, we found that compared to ovarian cancer cells grown in monolayers, ovarian cancer spheroids exhibited increased SMYD3 expression associated with higher levels of H3K4me3 (5). The SMYD3-H3K4me3-integrin pathway plays an important role in the pathogenesis of implantation metastasis in ovarian cancer. SMYD3-induced enhancement of spheroid invasion and adhesion is mediated by the downstream effectors ITGB6 and ITGAM (7). However, the potential mechanisms underlying SMYD3/integrin-mediated invasion and adhesion still need to be explored.

It is widely accepted that TGF $\beta$ plays a critical role in tumor progression and can mediate tumor immune escape, promote epithelial-mesenchymal transition (EMT) and help cells differentiate into more aggressive phenotypes for metastasis $(8,9)$. TGF $\beta 1$ is upregulated in ovarian cancer tissues and is associated with tumor progression, chemotherapy resistance and a poor prognosis (10). Nevertheless, more studies on the role of TGF $\beta 1$ in ovarian cancer metastasis are required.

TGF $\beta 1$ is secreted by cancer cells or fibroblasts in an inactive form, which consists of the $12.5-\mathrm{kDa}$ carboxy-terminal region of TGF $\beta 1$ and a $25-\mathrm{kDa}$ amino-terminal latency-associated peptide (LAP) joined by noncovalent bonding. This configuration prevents TGF $\beta 1$ from binding to its receptor (11). An acidic $\mathrm{pH}$, reactive oxygen species, various kinases, thrombospondin-1 (THBS-1) and shear stress can activate latent TGF $\beta 1(12,13)$. In a study on pulmonary fibrosis, ITGB6 was found to be the key activator of TGF $\beta 1$ in lung epithelial cells (14). According to reports and our research base, we speculated that SMYD3/ITGB6 promotes the invasion and adhesion of ovarian cancer spheroids by changing the configuration of TGF $\beta 1$-LAP and then activating TGF $\beta 1$.

In this report, we found that SMYD3 and ITGB6 could promote the release and activation of latent TGF $\beta 1$ and increase the phosphorylation of Smad3 activated by TGF $\beta 1$ in ovarian cancer spheroids. As downstream targets of the TGF $\beta 1 /$ SMAD3 pathway and essential participants in EMT promotion, $\mathrm{N}$-cadherin, Vimentin, E-cadherin and Snail were found to be regulated by SMYD3 and ITGB6 and could contribute to enhanced invasion and adhesion in ovarian cancer spheroids. In addition, TGF $\beta 1$ could also upregulate the expression of SMYD3 and ITGB6 in return to form a positive feedback loop with the SMYD3/ITGB6/TGF $\beta 1$ pathway to enhance the metastasis of ovarian cancer spheroids.

\section{MATERIALS AND METHODS}

\section{Cell Lines and Culture Conditions}

The human EOC cell line HEY was a gift from Dr. Robert Bast's laboratory at the University of Texas Anderson Cancer Center, Houston, TX. The human EOC cell line A2780 were obtained from the Shanghai Key Laboratory of Female Reproduction Endocrine Related Diseases, Obstetrics and Gynecology Hospital, Fudan University. HEY and A2780 cells were cultured in complete RPMI 1640 medium at $37^{\circ} \mathrm{C}$ in a $5 \% \mathrm{CO}_{2}$ environment. All complete media were supplemented with $10 \%$ fetal bovine serum (FBS), $100 \mathrm{U} / \mathrm{ml}$ penicillin and $100 \mu \mathrm{g} /$ $\mathrm{ml}$ streptomycin.

For adherent culture (2D culture), cells were cultured in 10cm common culture dishes (Corning, 430167) or common 6-well plates (Corning, 3516).

For suspended culture (3D culture), cells were cultured in 10$\mathrm{cm}$ ultralow-attachment culture dishes (Corning, 3262) or ultralow-attachment 6-well plates (Corning, 3471) for 3 days.

\section{siRNA-Mediated Gene Silencing}

A SMYD3-specific siRNA duplex (si-SMYD3, sequence: 5'CCACAAGCGGGAAUGCAAA-3') was synthesized by Genomeditech (Shanghai, China). NC-SMYD3 (5'UUCUCCGAACGUGUCACGUdTdT-3') was used as a negative control. An ITGB6-specific siRNA duplex (si-ITGB6, targeting the sequence 5'-GUCAAAGGAUGUCAAUUAATT-3') was synthesized by GenePharma (Shanghai, China). NC-ITGB6 (5'-UUCUCCGAACGUGUCACGUTT-3') was the corresponding negative control. An ITGAV-specific siRNA duplex (si-ITGAV, sequence: 5'-GAAUAUCGGUUGGAUU AUA-3') was synthesized by Genomeditech (Shanghai, China). NC-ITGAV (5'-UUCUCCGAACGUGUCACGUdTdT-3') was used as a negative control. For siRNA transfection, $1.5^{\star} 10^{5} \mathrm{HEY}$ cells/well or $3^{\star} 10^{5} \mathrm{~A} 2780$ cells/well were seeded in a 6-well plate. The next day, we used Lipofectamine 3000 reagent (Invitrogen) for transient transfection according to the manufacturer's protocol. Six to twelve hours later, the cells were trypsinized, counted and seeded in 2D and 3D culture systems for further experiments. 


\section{Western Blotting}

Antibodies against SMYD3 (Abcam, ab817149), ITGB6 (Abcam, ab187155), Smad3 (Abcam, ab40854), p-Smad3 (Abcam, ab52903), Snail (Cell Signaling Technology (CST), C15D3), Ncadherin (Abcam, ab76011), E-cadherin (CST, 3195), Vimentin (CST, 5741) and GAPDH (CST, 14C10) were used for western blotting. Cells were lysed in RIPA buffer containing 1:100 PMSF. A BCA protein assay kit (Solarbio, P0020) was used to measure the protein concentration. Equal amounts of protein were resolved by SDS-PAGE, transferred to PVDF membranes, and incubated with appropriate primary antibodies at the indicated concentration. Immune complexes were detected with HRPconjugated secondary antibodies (Arigo Biolaboratories, ARG65351) and ECL chemiluminescence reagent (EpiZyme, SQ201). Each western blotting experiment was repeated at least two to three times. We quantified the western blot bands using ImageJ software.

\section{Cell Treatments Using an Inhibitor of and an Antibody Against TGF $\beta 1$}

Cells were seeded in ultralow-attachment 6-well plates. For active TGF $\beta 1$ and SB431542 inhibitor treatment, $10 \mathrm{ng} / \mathrm{ml}$ recombinant human TGF $\beta 1$ (rhTGF $\beta 1$ ) protein (Abcam, ab50036) was added to the culture medium for $72 \mathrm{~h}$, and the medium was replaced at $48 \mathrm{~h}$. Then, $10 \mu \mathrm{M}$ SB431542 (MedChemExpress, HY-10341) was added to the medium and incubated for $6 \mathrm{~h}$ (equal doses of PBS and DMSO were used as the negative controls for rhTGF $\beta 1$ and SB431542, respectively). The cells were collected for further western blotting and cell function assays. For the anti-ITGB6 inhibition assay, $10 \mu \mathrm{g} / \mathrm{ml}$ anti-ITGB6 antibody (Millipore, MAB2076Z) was added to the culture medium as a pretreatment to block ITGB6 for $12 \mathrm{~h}$. Then, $10 \mathrm{ng} / \mathrm{ml}$ latent TGF $\beta 1$ (CST, 5154) was added to the culture medium for $72 \mathrm{~h}$ (equal doses of normal mouse IgG1 (Santa Cruz Biotechnology, sc-3877) and PBS were used as the negative controls for the anti-ITGB6 antibody and latent TGF $\beta 1$ experiments, respectively). The cells were collected for further western blotting and cell function assays.

\section{Transwell Invasion Assay}

A Transwell system (24-well insert, pore size: $8 \mathrm{~mm}$, Corning, 3422 ) was used to measure the invasive ability of $3 \mathrm{D}$-cultured HEY cells. The inserts were coated with $50 \mu \mathrm{l}$ of Matrigel (BD Bioscience Pharmingen, 356234) at a 1:8 dilution and incubated at $37^{\circ} \mathrm{C}$ overnight. The following day, cells were trypsinized, counted and resuspended in serum-free medium. A total of $1^{\star} 10^{4}$ cells/200 $\mu \mathrm{l}$ of serum-free medium were added to the upper well of the chamber. In addition, $600 \mu \mathrm{l}$ of complete medium was added to the lower well. After incubation for $16 \mathrm{~h}$, the upper surface of the membrane was wiped with cotton swabs to remove any remaining cells, and the cells on the lower surface of the membrane were fixed with $4 \%$ paraformaldehyde and stained with $2 \%$ crystal violet. Five representative fields of each insert were imaged and counted using an Olympus light microscope at $100 \times$ magnification.

\section{Adhesion Assay}

A 24-well plate was coated with $200 \mu \mathrm{l}$ of Matrigel at a 1:50 dilution and air-dried in a biosafety cabinet for $6 \mathrm{~h}$. Then, for blocking non-specific binding, $200 \mu \mathrm{l}$ of serum-free medium containing $0.1 \%$ BSA (bovine serum albumin, Mpbio, 02FC007710) for $1 \mathrm{~h}$. 3D-cultured cells were trypsinized, counted and resuspended in serum-free medium. A total of $3^{\star} 10^{4}$ cells $/ 200 \mu \mathrm{l}$ of serum-free medium were added to each Matrigel-coated well and incubated in the incubator for 1-2 h. Nonadherent cells were removed by washing with PBS. Adherent cells were fixed with $4 \%$ paraformaldehyde and stained with $2 \%$ crystal violet. Five representative fields of each insert were imaged and counted using an Olympus light microscope at $40 \times$ magnification.

\section{D Tumor Spheroid Invasion Assay}

This assay was performed as previously described (15). HEY cells $\left(2 \times 10^{4}\right.$ cells $\left./ \mathrm{ml}\right)$ were plated in ultralow-attachment 96 -well round-bottomed plates in $200 \mu \mathrm{l}$ of medium/well. rhTGF $\beta 1$ protein $(10 \mathrm{ng} / \mathrm{ml})$ was added to treat the cells in the TGF $\beta 1+\mathrm{DMSO}$ and TGF $\beta 1+$ SB431542 groups. The same volume of PBS was added to treat cells in the PBS+DMSO and PBS+SB431542 groups. After 3 days of incubation, $100 \mu \mathrm{l}$ of culture medium was gently removed from each well, and $100 \mu \mathrm{l}$ of Matrigel matrix was gently dispensed into each bottom well. The plate was transferred into a $37^{\circ} \mathrm{C}$ incubator, and the Matrigel matrix, which contained $20 \mathrm{ng} / \mathrm{ml}$ rhTGF $\beta 1$ or $20 \mu \mathrm{M}$ SB431542 according to the experimental design, was allowed to solidify. One hour later, $100 \mu \mathrm{l}$ of serum-free growth medium containing $10 \mathrm{ng} /$ $\mathrm{ml}$ rhTGF $\beta 1$ or $10 \mu \mathrm{M}$ SB431542 was gently added to each well. An image was recorded for each tumor spheroid at 0,24 , and $48 \mathrm{~h}$ to dynamically observe the $3 \mathrm{D}$ tumor spheroid invasion ability. Representative fields of spheroids were randomly counted using an Olympus light microscope at $100 \times$ magnification. The diameters of the spheroids were measured by ImageJ software.

\section{Dual-Luciferase Reporter Assay}

A fragment of the PAI-1 promoter (-799 71 bp) was amplified and cloned into the pGL3 vector, which contains firefly luciferase (pGL3-PAI-1). Luciferase activity was measured using a dualluciferase reporter assay system at $24 \mathrm{~h}$ posttransfection according to the manufacturer's instructions (Promega, E1910). Normalized data were calculated as the ratio of firefly/ renilla luciferase activities.

\section{ELISA}

The TGF $\beta 1$ concentration in the supernatant was measured with a Quantikine ELISA Human TGF $\beta 1$ kit (R\&D Systems). A2780 cells were first subjected to siRNA-mediated gene silencing treatment for $48 \mathrm{~h}$. A total of $6 \times 10^{5}$ cells were suspended in $1.5 \mathrm{ml}$ of culture medium, added to a single well of a six-well ultralow-attachment plate, and then cultured for $72 \mathrm{~h}$. The culture medium was not changed during the culture period. Then, the culture medium was collected in a $1.5 \mathrm{ml}$ conical tube and centrifuged at 3,000 rpm for 15 minutes. The concentration of TGF $\beta 1$ in the culture medium was determined using a human 
TGF $\beta 1$ Quantikine ELISA kit (R\&D Systems). $\mathrm{HCl}$ and $\mathrm{NaOH} /$ HEPES were used to convert latent TGF $\beta 1$ into the activated form.

\section{Immunofluorescence Assay}

Hey and A2780 spheroids were embedded with Matrigel matrix in confocal dishes. The cells were fixed with $4 \%$ formaldehyde for $24 \mathrm{~h}$, and blocked with $4 \%$ goat serum for $1 \mathrm{~h}$ at room temperature. The cells were then incubated with antibodies against E-cadherin (CST, 3195, 1:200), N-cadherin (CST, 13116, 1:200), at $4^{\circ} \mathrm{C}$ overnight. The dishes and slides were washed and incubated with Alexa Fluor 594 AffiniPure Donkey Anti- Rabbit IgG (H+L) (Yeasen, 34212ES60, 1:100) for $1 \mathrm{hr}$ at room temperature. For F-actin, the cells were treated with $0.5 \%$ Triton (Absin, abs47048168) for $5 \mathrm{~min}$ and then, were incubated with Phalloidin (Yeasen, 40734ES75, 1:200) for $30 \mathrm{~min}$ at room temperature. Finally, the dishes were stained with DAPI (Beyotime, C1002, 1:1000) for $5 \mathrm{~min}$. The fluorescently labeled cells (dishes and slides) were examined under a confocal laser scanning microscope (Zeiss LSM 880 Confocal Microscope, Germany) at room temperature.

\section{Statistical Analysis}

Statistical analysis and graphing were conducted using GraphPad Prism 8. ImageJ was used for protein quantification. Statistically significant differences were determined by Student's t-test, and $\mathrm{p}$ values $<0.05$ were considered significant.

\section{RESULTS}

\section{Key EMT Factors Are Promoted Along With SMYD3 and ITGB6 Upregulation During Ovarian Cancer Cell Spheroid Formation}

According to our previous study, SMYD3 is overexpressed in ovarian cancer ascites spheroids compared with primary ovarian cancer tissues. Using a 3D culture model to mimic the suspended growth conditions in ascites, we found that SMYD3 could enhance the adhesion and invasion of ovarian cancer spheroids and promote metastasis of ovarian cancer in vivo by upregulating the expression of ITGB6 and ITGAM (7). Compared with 2Dcultured HEY and A2780 cells, the corresponding 3D-cultured cells showed higher expression of SMYD3 and ITGB6, which indicated that the ovarian cancer spheroids had a more invasive phenotype. In addition, we found that the expression of $\mathrm{N}$ cadherin, Snail, Vimentin and E-cadherin, which are essential molecules in the EMT process, was also increased in $3 \mathrm{D}$-cultured HEY and A2780 cells (Figure 1A). Tumor cells can be more invasive during the EMT process. With this in mind, what is the mechanism of the upregulation of N-cadherin, Snail, and Vimentin and downregulation of E-cadherin during 2Dcultured ovarian cancer cell transformation into 3D spheroids? Do these EMT-related genes contribute to SMYD3/ITGB6mediated spheroid metastasis? Both these questions need to be answered.
A



B
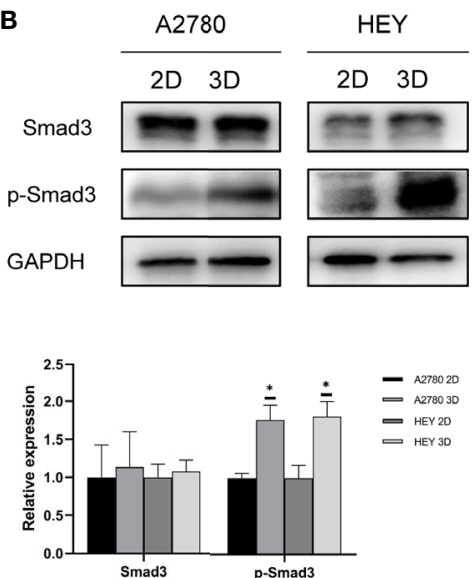

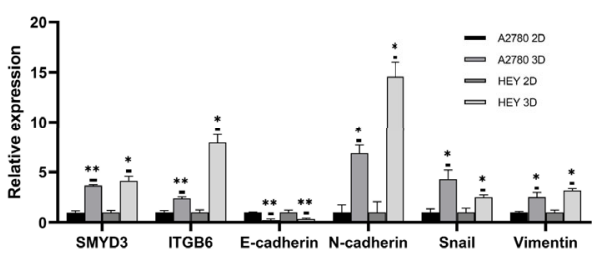

FIGURE 1 | EMT along with SMYD3 and ITGB6 upregulation is promoted during ovarian cancer cell spheroid formation. (A) The expression levels of SMYD3, ITGB6, N-cadherin, Snail, Vimentin and E-cadherin in 2D- and 3D-cultured HEY and A2780 cells were evaluated by western blot analysis. (B) The phosphorylation level of Smad3 in 2D- and 3D-cultured HEY and A2780 cells was evaluated by western blot analysis. In the histogram of the western blot quantification, "*" refers to $p<0.05$ and "**" refers to $p<0.01$. 
Smad3, as a well-known downstream signal of TGF $\beta 1$, can be activated by phosphorylated ALK5 after binding of TGF $\beta 1$ to its receptor (16). Since the TGF $\beta 1 / \mathrm{Smad} 3$ signal transduction pathway is involved in inducing EMT in ovarian cancer (17), we aimed to identify whether the TGF $\beta 1 / \mathrm{Smad} 3$ pathway is more activated in $3 \mathrm{D}$-cultured ovarian cancer spheroids than in 2D-cultured ovarian cancer cells. As shown in Figure 1B, higher phosphorylation levels of Smad3 were found in 3D-cultured HEY and A2780 cells than in 2D-cultured cells, which indicated that TGF $\beta 1 / \mathrm{Smad} 3$ might play a role in promoting the EMT process in ovarian cancer spheroids.

\section{Activation of the TGF $\beta 1 /$ Smad3 Pathway Is Conducive to the Regulation of EMT-Related Genes in 3D-Cultured Ovarian Cancer Spheroids}

A review of the previous literature about the induction of EMT by TGF $\beta 1$ showed that the epithelial markers E-cadherin was repressed while the mesenchymal markers Vimentin and $\mathrm{N}$ cadherin were induced during the induction of EMT by TGF $\beta 1$ (18). Since phosphorylated Smad3, N-cadherin, Snail and Vimentin were all increased in 3D-cultured ovarian cancer spheroids and E-cadherin was decreased in 3D-cultured ovarian cancer spheroids, we aimed to determine whether $\mathrm{N}$ cadherin, Snail, Vimentin and E-cadherin are target molecules of the activated Smad3 pathway in 3D-cultured ovarian cancer spheroids. First, we used rhTGF $\beta 1$ to treat $3 \mathrm{D}$-cultured HEY and A2780 cells for 24, 48 and 72 hours. Over time, the amount of phosphorylated Smad3 increased under the condition of constant expression of total Smad3, which demonstrated that TGF $\beta 1$ could stimulate the Smad3 pathway. In addition, the expression of $\mathrm{N}$-cadherin, Snail and Vimentin was also increased and the expression of E-cadherin was decreased after treatment with rhTGF $\beta 1$ (Figure 2A). Subsequently, SB431542, an inhibitor of ALK5, was added to the supernatant of 3Dcultured HEY and A2780 cells and incubated for 6 hours. As the phosphorylation of Smad3 was inhibited by SB431542, the expression of $\mathrm{N}$-cadherin, Snail and Vimentin also declined, and the expression of E-cadherin was enhanced (Figure 2B). Furthermore, we found that the regulation of EMT-related genes induced by rhTGF $\beta 1$ could be restrained by SB431542 (Figure 2C). These findings showed that TGF $\beta 1$ could stimulate the Smad3 pathway and contribute to the increases in the expression of Snail and N-cadherin. Finally, we used Transwell invasion, adherence and 3D tumor spheroid invasion assays to validate the functional effects of rhTGF $\beta 1$ and SB431542 on the EMT process. RhTGF $\beta 1$ enhanced the invasion and adhesion of 3D-cultured HEY cells, and these effects were inhibited when SB431542 was added simultaneously (Figures 2D, E).

\section{SMYD3 and ITGB6 Can Activate the TGFB1/Smad3 Pathway and Regulate the Expression of EMT-Related Genes in 3D-Cultured Ovarian Cancer Spheroids}

To determine why SMYD3 and ITGB6 could make ovarian cancer spheroids more invasive, we downregulated the expression of
SMYD3 and ITGB6 in 3D-cultured HEY and A2780 cells. As shown in Figure 3A, after SMYD3 silencing, the expression of its target gene ITGB6 was also decreased. In addition, the phosphorylation levels of Smad3 were consequently reduced, along with decreased expression of $\mathrm{N}$-cadherin, Snail, and Vimentin and increased expression of E-cadherin. After ITGB6 silencing, the expression changes in p-Smad3, N-cadherin, Snail, Vimentin, Ecadherin were consistent with those observed with SMYD3 silencing (Figure 3B). In addition, using immunofluorescence assay, we also demonstrated that when we inhibited the expression of SMYD3 and ITGB6, the expression of E-cadherin significantly increased. And as the 3D-cultured ovarian cells were treated with $\operatorname{rhTGF} \beta 1$, it significantly decreased. The expression of $\mathrm{N}$-cadherin showed opposite tendency to the one of E-cadherin (Figures 3C, D).

Considering to the expression changes of EMT-related genes, we should also pay attention to the morphological changes of 3D-cultured ovarian cancer cells. The epithelial-like structure of $3 \mathrm{D}$-cultured spheroid is more solid round. And the mesenchymal-like structure of $3 \mathrm{D}$-cultured is grape-like or spindle like (19). The 3D-cultured HEY and A2780 spheroid with SMYD3 or ITGB6 silencing presented the robust cell-cell adherence and more solid structure compared with 3D-cultured HEY and A2780-NC spheroid (Figure 3C). The 3D-cultured HEY and A2780 spheroid presented the loose cell-cell adherence and spindle-like structure when it was exposed to rhTGF $\beta 1$ (Figure 3D). Therefore, SMYD3 and ITGB6 could stimulate the TGF $\beta 1 /$ Smad3 pathway and regulate the expression of $\mathrm{N}$ cadherin, Snail, Vimentin, E-cadherin in ovarian cancer spheroids and promote the EMT process.

\section{Latent TGF $\beta 1$ Can Bind to ITGB6 and Release Active TGF $\beta 1$ to Stimulate the Smad3 Pathway}

How can SMYD3 and ITGB6 stimulate the TGF $\beta 1 / \mathrm{Smad} 3$ pathway? TGF $\beta 1$ is produced as a latent precursor and functions in an active form. Therefore, the activation of latent TGF $\beta 1$ is a crucial regulatory event. ITGB6 was reported to be important in stringently localized activator of latent TGF $\beta 1$ at epithelial surfaces (20). Therefore, we performed a dualluciferase reporter assay to identify whether ITGB6 plays a role in the activation of TGF $\beta 1$. Plasminogen activator inhibitor-1 (PAI-1) is a well-known target gene of TGF $\beta 1$ (21). When we downregulated the expression of ITGB6 in 3D-cultured HEY cells, the relative luciferase activity of PAI-1 was decreased accordingly $(\mathrm{p}<0.0001)$ (Figure $4 \mathbf{A})$. These results suggested that ITGB6 could stimulate the TGF $\beta 1$ pathway. Then, we used latent TGF $\beta 1$ to treat 3D-cultured HEY and A2780 cells. As a result, the Smad3 pathway was activated. However, after adding a monoclonal antibody against ITGB6 to inhibit latent TGF $\beta 1$ binding to ITGB6, latent TGF $\beta 1$ failed to stimulate the Smad3 pathway (Figure 4B). Therefore, latent TGF $\beta 1$ could be transformed into the active form by binding to ITGB6 and then induce the activation of the Smad3 pathway.

As the binding partner of ITGB6, ITGAV has also been reported to be able to activate latent TGF $\beta 1$ in tumors $(22,23)$. It is necessary to investigate whether ITGAV could regulate the 
A

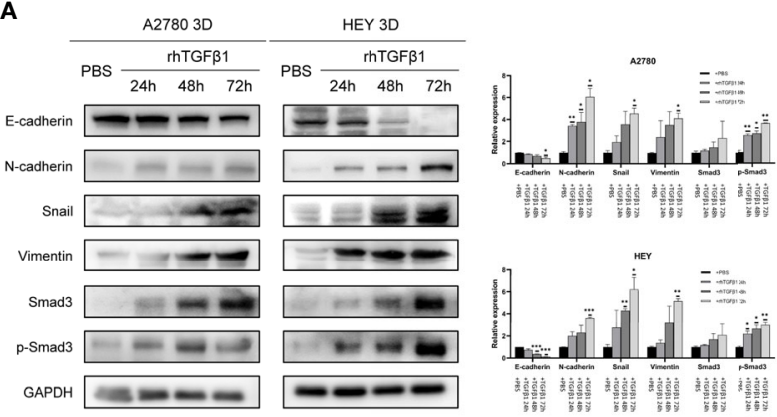

B
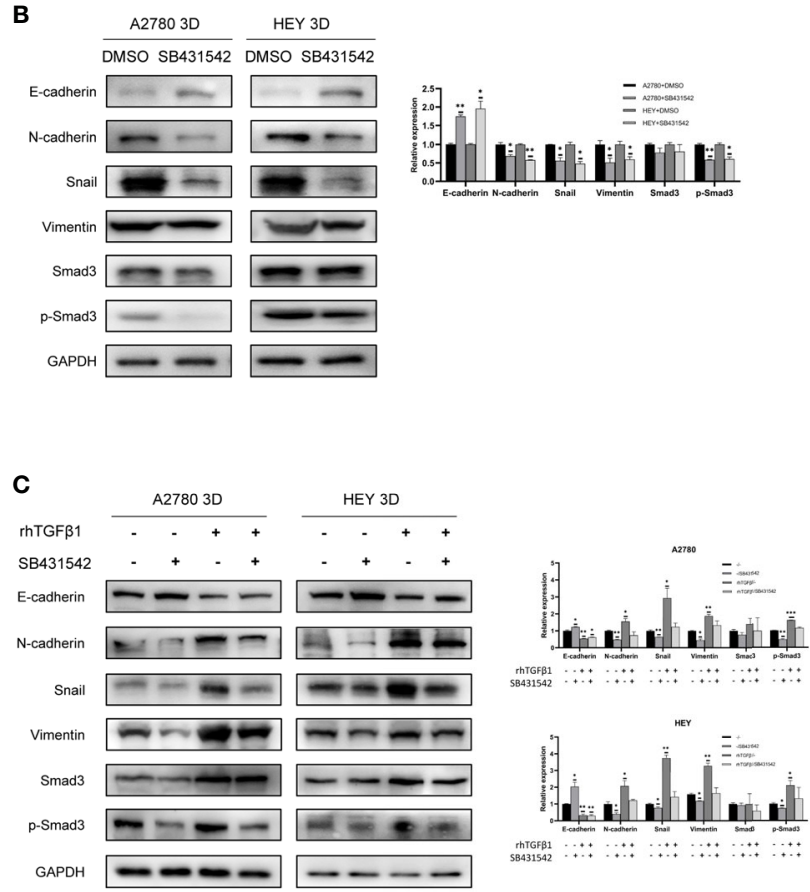

D



FIGURE 2 | Continued 


\begin{abstract}
FIGURE 2 | Activation of the TGF $\beta 1 / S m a d 3$ pathway is conducive to the upregulation of EMT-related genes in 3D-cultured ovarian cancer spheroids. (A) Changes in the expression of $\mathrm{N}$-cadherin, Snail, Vimentin and E-cadherin and the phosphorylation level of Smad3 in 3D-cultured HEY and A2780 cells after treatment with 10 ng/ml rhTGF $\beta 1$ protein for 24, 48 and 72 h (B) Changes in the expression of N-cadherin, Snail, Vimentin and E-cadherin and the phosphorylation level of Smad3 in 3D-cultured HEY and A2780 cells after treatment with $10 \mu \mathrm{M}$ SB431542 for 6h. (C) Changes in the expression of N-cadherin, Snail, Vimentin and E-cadherin and the phosphorylation level of Smad3 in 3D-cultured HEY and A2780 cells after treatment with or without rhTGF 31 and SB431542. RhTGF $\beta 1$ protein (10 ng/ml) was added to the culture medium for $72 \mathrm{~h}$ Then, $10 \mu \mathrm{M}$ SB431542 was added to the medium and incubated for $6 \mathrm{~h}$ (equal doses of PBS and DMSO were used as the negative controls for rhTGF $\beta 1$ and SB431542, respectively). (D) Transwell invasion and adhesion assays revealed changes in the invasion and adhesion of 3D-cultured HEY cells after treatment with or without rhTGF $\beta 1$ and SB431542. (E) 3D tumor spheroid invasion assays revealed changes in the invasion and adhesion of 3D-cultured HEY cells after treatment with or without rhTGF $\beta 1$ and SB431542. RhTGF $\beta 1$ protein (10 $\mathrm{ng} / \mathrm{ml})$ was added to treat cells in the rhTGF $\beta 1+D M S O$ and rhTGF $\beta 1+$ SB431542 groups. The same volume of PBS was added to treat cells in the PBS+DMSO and PBS+SB431542 groups. After 3 days of incubation, $100 \mu$ l of culture medium was gently removed from each well, and $100 \mu$ of Matrigel matrix was gently dispensed into each bottom well. Matrigel matrix containing 20 ng/ml rhTGF $\beta 1$ was used in the rhTGF $\beta 1+D M S O$ and rhTGF $\beta 1+$ SB431542 groups, and $20 \mu \mathrm{M}$ SB431542 was used in the rhTGF $\beta 1+$ SB431542 and PBS+SB431542 groups. One hour later, $100 \mu$ l of serum-free growth medium containing $10 \mathrm{ng} / \mathrm{ml}$ rhTGF $\beta 1$ was gently added into each well of the rhTGF $\beta 1+$ DMSO and rhTGF $\beta 1+$ SB431542 groups. SB431542

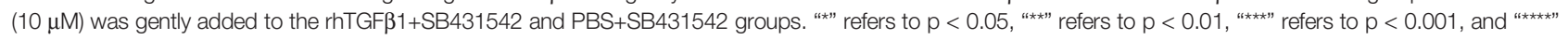
refers to $p<0.0001$.
\end{abstract}

activation of latent TGF $\beta 1$ in $3 \mathrm{D}$-cultured ovarian cancer spheroids and whether ITGAV is involved in the ITGB6regulated activation of latent TGF $\beta 1$ in $3 \mathrm{D}$-cultured ovarian cancer spheroids. First, we silenced the expression of ITGAV in 3D-cultured ovarian cancer spheroids using siRNA. After treatment with latent TGF $\beta 1$, the phosphorylation level of Smad3 did not decrease in ITGAV knockdown cells. Therefore, ITGAV might have no effect on the activation of latent TGF $\beta 1$ in 3D-cultured ovarian cancer spheroids. Then, we used $10 \mu \mathrm{g} / \mathrm{ml}$ anti-ITGB6 antibody to block ITGB6 for $12 \mathrm{~h}$ in 3D-cultured NCITGAV and si-ITGAV cells. After that, $10 \mathrm{ng} / \mathrm{ml}$ latent TGF $\beta 1$ was added to the culture medium for $72 \mathrm{~h}$. We found that antiITGB6 antibody was more effective in inhibiting the phosphorylation level of Smad3 in 3D-cultured NC-ITGAV cells than in 3D-cultured si-ITGAV cells, implying that ITGAV might play a role in ITGB6-regulated activation of latent TGF $\beta 1$ in 3Dcultured ovarian cancer spheroids (Figure 4C).

\section{SMYD3 and ITGB6 Can Facilitate the Release of Latent TGF $\beta 1$ From 3D-Cultured Ovarian Cancer Spheroids}

Since we found that SMYD3 and ITGB6 could upregulate the expression of Snail and N-cadherin via the activation of the TGF $\beta 1 /$ Smad3 pathway, whether SMYD3 and ITGB6 can enhance the release of latent TGF $\beta 1$ needed to be explored. We used ELISA to quantify the amounts of active and total TGF $\beta 1$, and the difference was considered the amount of latent TGF $\beta 1$. We found that the latent TGF $\beta 1$ level was decreased after downregulating SMYD3 or ITGB6 (both $\mathrm{p}<0.0001$ ) (Figures 5A, B). Hence, SMYD3 and ITGB6 could not only activate latent TGF $\beta 1$ but also increase the release of latent TGF $\beta 1$ from 3D-cultured ovarian cancer spheroids.

\section{TGF $\beta 1$ Can Promote the Expression of SMYD3 and ITGB6 via Feedback}

We verified that ITGB6 could activate latent TGF $\beta 1$. Subsequently, we found that TGF $\beta 1$ could also promote the expression of SMYD3 and ITGB6 in 3D-cultured HEY and A2780 cells (Figure 6A). SB431542 reduced the expression of SMYD3 and ITGB6 (Figure 6B). The upregulation of SMYD3 and ITGB6 due to the activation of TGF $\beta 1$ could be inhibited by SB431542 (Figure 6C). These findings demonstrated that TGF $\beta 1$ promotes the expression of SMYD3 and ITGB6 via the Smad3 pathway. As shown in Figure 6D, latent TGF $\beta 1$ facilitated the expression of SMYD3 and ITGB6. After the binding of latent TGF $\beta 1$ to ITGB6 was blocked, the expression of SMYD3 and ITGB6 also declined. Thus, the SMYD3/ITGB6/TGF $\beta 1$ positive feedback loop drove the upregulation of Snail and N-cadherin, which promoted invasion, adhesion and EMT progression during ovarian cancer metastasis.

\section{DISCUSSION}

Ovarian cancer is the leading cause of death among gynecological malignancies. Worldwide, the incidence rate of ovarian cancer is seventh among those of female malignant cancers, while the mortality rate is eighth, and the 5-year survival rate is below 45\% (24). The main metastatic mode of ovarian cancer is the transcoelomic pathway. Briefly, cells disseminate from the primary epithelial ovarian tumor and float as spheroids in the ascites. Metastatic cells then attach to peritoneal organs and form macrometastatic colonies (25). Both genetic changes and epigenetic changes contribute to the initiation and transcoelomic process of ovarian cancer. This disease is characterized by "genomic chaos" caused by high chromosomal instability due to a massive number of copy number abnormalities and chromosomal alterations $(26,27)$. Therefore, epigenetic enzymes may play a great role in regulating the transcoelomic spread of ovarian cancer.

According to the previous work of our team, SMYD3, a histone methylation transferase, was found to be expressed at higher levels in ascites-derived spheroids than in primary ovarian tumor cells from EOC patients. In ovarian cancer spheroids, SMYD3 was found bind to H3K4me3 at the ITGB6 and ITGAM promoter regions to upregulate the expression of ITGB6 and ITGAM, which was verified to enhance the invasion and adhesion of ovarian cancer spheroids (7). ITGB6 is an essential member of the integrin family. The integrin family is documented to be involved in various cancer processes (such as tumor initiation, metastasis and drug resistance), and ITGB6 is also reported to be associated with the progression and metastasis of oral squamous cell carcinoma, bladder cancer and colorectal carcinoma (28-31). Previously, we found that 
A

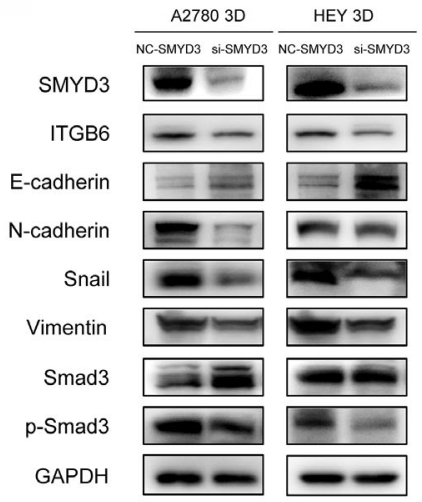

B

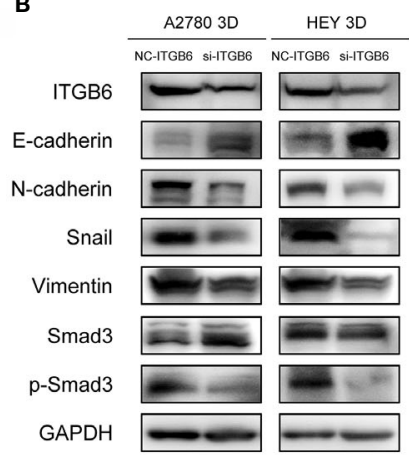

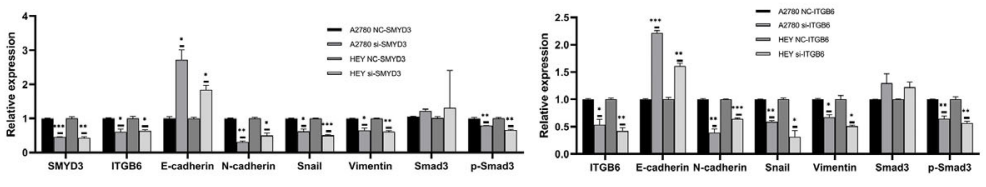

C



HEY


si-ITGB6


+ rhTGFß1
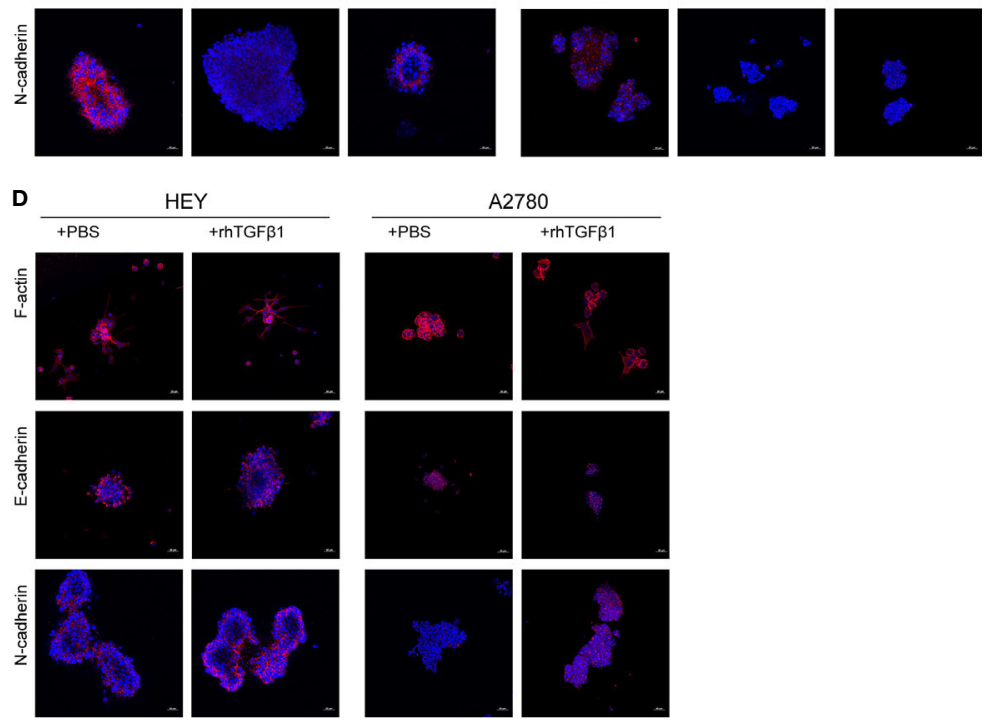

FIGURE 3 | SMYD3 and ITGB6 can activate the TGF31/Smad3 pathway and upregulate the expression of EMT-related genes in 3D-cultured ovarian cancer spheroids. (A) The changes in the phosphorylation level of Smad3 and the expression of SMYD3, ITGB6, N-cadherin, Snail, Vimentin and E-cadherin in 3D-cultured HEY and A2780 cells were evaluated by western blot analysis after SMYD3 silencing. (B) The changes in the phosphorylation level of Smad3 and the expression of ITGB6, $\mathrm{N}$-cadherin, Snail, Vimentin and E-cadherin in 3D-cultured HEY and A2780 cells were evaluated by western blot analysis after ITGB6 silencing. (C) Immunofluorescence assay exhibited the expression changes of F-actin, E-cadherin and N-cadherin in both 3D-cultured HEY and A2780 cells after silencing SMYD3 and ITGB6, respectively. (D) Immunofluorescence assay exhibited the expression changes of F-actin, E-cadherin and N-cadherin in both 3D-cultured HEY and A2780 cells after rhTGF 31

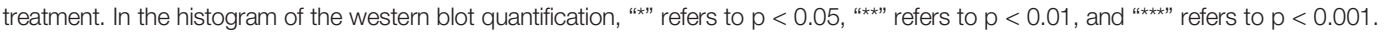


A

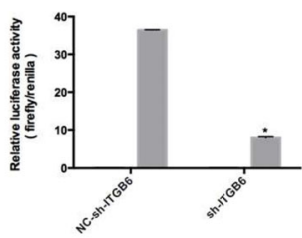

B
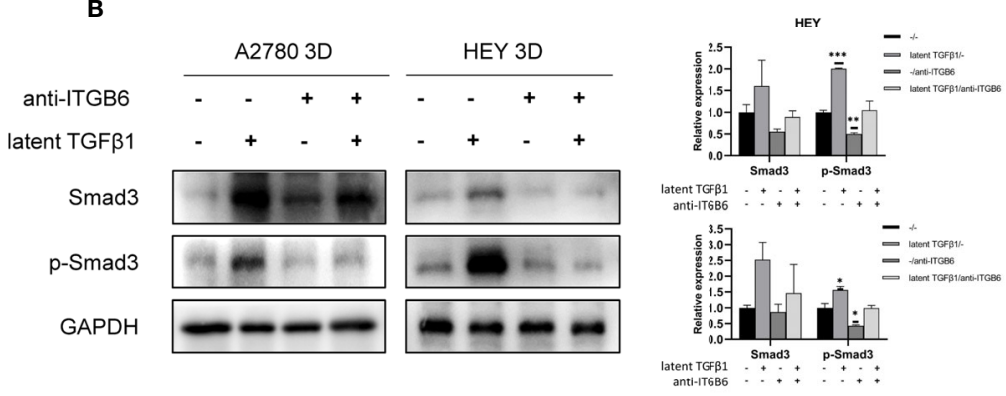

C

A2780 3D NC-ITGAV si-ITGAV

anti-ITGB6

ITGAV

Smad3

P-Smad3

GAPDH


HEY 3D NC-ITGAV Si-ITGAV
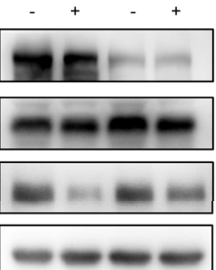

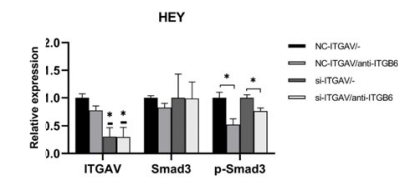

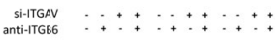

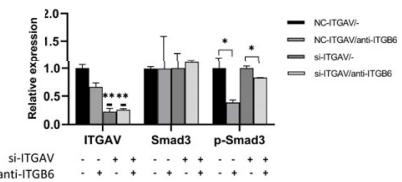

FIGURE 4 | Latent TGF $\beta 1$ can bind to ITGB6 and release active TGF $\beta 1$ to stimulate the Smad3 pathway. (A) A dual-luciferase reporter assay showed changes in 3D-cultured HEY cells after ITGB6 silencing. (B) Changes in the phosphorylation level of Smad3 in 3D-cultured HEY and A2780 cells after treatment with or without latent TGFB1 and anti-ITGB6 antibody. Anti-ITGB6 antibody $(10 \mu \mathrm{g} / \mathrm{ml})$ was added to the culture medium as a pretreatment to block ITGB6 for $12 \mathrm{~h}$. Then, $10 \mathrm{ng} / \mathrm{ml}$ latent TGF $\beta 1$ was added to the culture medium for $72 \mathrm{~h}$ (equal doses of normal mouse IgG1 and PBS were used as the negative controls for the anti-ITGB6 antibody and latent TGF $\beta 1$, respectively). (C) Changes in the phosphorylation level of Smad3 in 3D-cultured HEY and A2780 cells after silencing of ITGAV. NC-ITGAV and si-ITGAV were transfected into HEY and A2780 cells. After $6 \mathrm{~h}$, the cells were resuspended in ultralow-attachment plates for 3D culture. Then, $10 \mu \mathrm{g} / \mathrm{ml}$ antiITGB6 antibody was used to block ITGB6 for $12 \mathrm{~h}$ in 3D-cultured NC-ITGAV and si-ITGAV cells, and equal doses of normal mouse IgG1 were used as a negative control. Subsequently, $10 \mathrm{ng} / \mathrm{ml}$ latent TGF $\beta 1$ was added to the culture medium and incubated for $72 \mathrm{~h}$ in each group. In the histogram of the quantification, "*”"

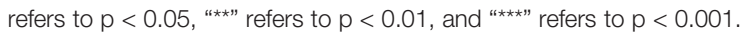

A

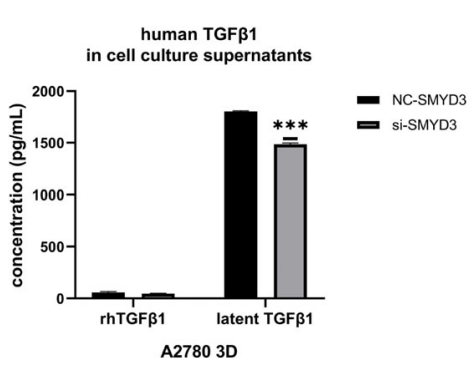

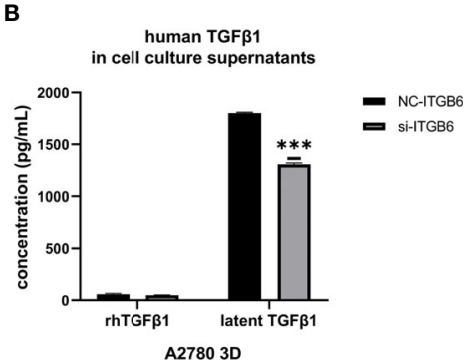

FIGURE 5 | SMYD3 and ITGB6 can facilitate the release of latent TGFB1 from 3D-cultured ovarian cancer spheroids. (A, B) ELISA was used to measure the concentrations of active TGF $\beta 1$ and latent TGF $\beta 1$ released from 3D-cultured A2780 cells after SMYD3 (A) or ITGB6 (B) silencing. In the histogram of the ELISA

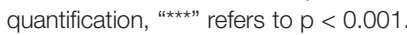


A

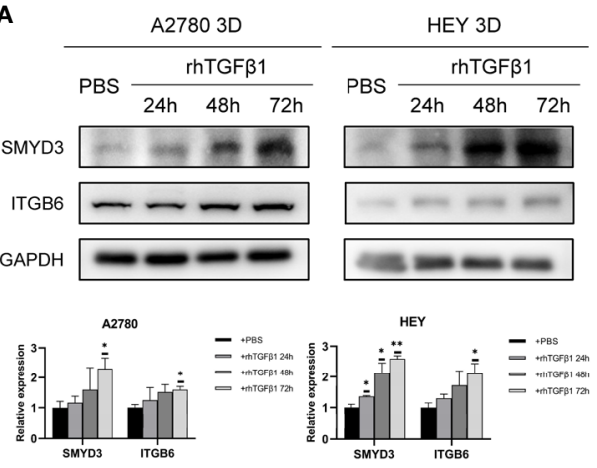

C


B

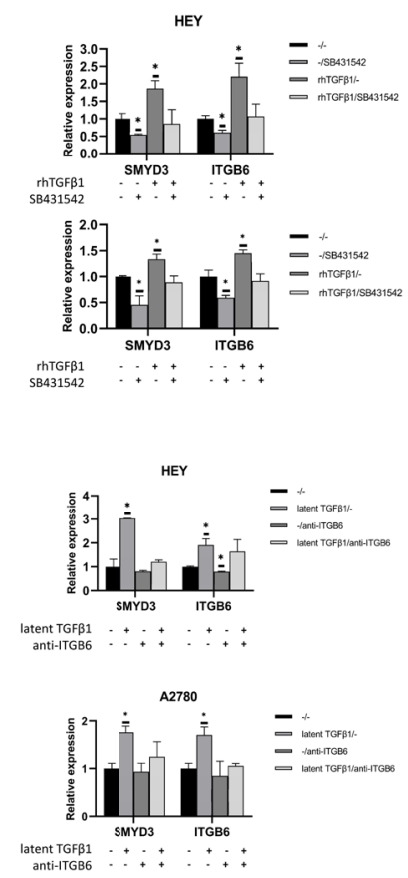

FIGURE 6 | TGF 31 can promote the expression of SMYD3 and ITGB6 via feedback. (A) Changes in the expression of SMYD3 and ITGB6 in 3D-cultured HEY and A2780 cells after treatment with $10 \mathrm{ng} / \mathrm{ml}$ recombinant human TGF 31 protein for 24, 48 and $72 \mathrm{~h}$. (B) Changes in the expression of SMYD3 and ITGB6 in 3Dcultured HEY and A2780 cells after treatment with $10 \mu \mathrm{M}$ SB431542 for 24, 48 and $72 \mathrm{~h}$. (C) Changes in the expression of SMYD3 and ITGB6 in 3D-cultured HEY and A2780 cells after treatment with or without rhTGF $\beta 1$ and SB431542. RhTGF 1 protein (10 $\mathrm{ng} / \mathrm{ml})$ was added to the culture medium and incubated for $72 \mathrm{~h}$. Then, $10 \mu \mathrm{M} / \mathrm{ml} \mathrm{SB} 431542$ was added to the medium and incubated for $6 \mathrm{~h}$ (equal doses of PBS and DMSO were used as the negative controls for rhTGF $\beta 1$ and SB431542, respectively). (D) Changes in the protein expression of SMYD3 and ITGB6 in 3D-cultured HEY and A2780 cells after treatment with or without latent TGF $\beta 1$ and anti-ITGB6. In the histogram of the western blot quantification, "*” refers to $p<0.05$ and "**" refers to $p<0.01$.

ITGB6 could promote the invasion and adhesion of ovarian cancer spheroids. However, the underlying molecular mechanisms should be further explored.

EMT is one of the phenotypic plasticity processes relevant to metastasis. During EMT, epithelial cells lose polarity and develop a stromal phenotype, increasing invasiveness. Cells with features of EMT are able to migrate, invade and undergo metastatic dissemination. TGF $\beta$ is a common inducer of the EMT process (32). TGF $\beta$ signaling promotes EMT by inducing the expression of several pleiotropic transcription factors, also known as "master regulators" of EMT (such as Snail, N-cadherin, Ecadherin and Vimentin). Proteins of the Smad family are the major effectors that control the transduction of intracellular signaling initiated by the TGF $\beta$ superfamily of cytokines. SMAD-dependent signaling can be activated by an active TGF $\beta 1$ ligand initially binding to TGF $\beta$ RII, followed by recruitment of ALK5 (TGFßRI) to the plasma membrane (33). Here, we observed that in ovarian cancer spheroids, the Smad3 
pathway was activated by TGF $\beta 1$ and upregulated the expression of Snail and N-cadherin, which are crucial genes in the EMT process.

The three isoforms of TGF $\beta$ (TGF $\beta 1$, TGF $\beta-2$ and TGF $\beta-3$ ) are always produced as inactive cytokines that cannot bind to their receptor and are not functional unless they are activated. An integrin-mediated mechanism makes an essential contribution to TGF $\beta$ activation in vivo. TGF $\beta$ activation has been demonstrated to be caused by the RGD tripeptide motif in the LAP region of latent TGF $\beta$ binding to specific integrin receptors (20). Khalid Puthawala et al. reported that integrin alpha(v)beta6-mediated TGF $\beta$ activation is required for radiation-induced lung fibrosis (34). Laura L Koth et al. found that integrin alpha(v)beta6-mediated TGF $\beta$ activation regulates the homeostasis of phospholipids and collectins in the lungs (35). To date, few articles have stated the role of ITGB6-mediated TGF $\beta$ activation in tumor progression. In our study, we found that downregulated expression of ITGB6 could reduce the activation level of the Smad3 pathway when ovarian cancer spheroids were treated with $\operatorname{rhTGF} \beta 1$. When ovarian cancer spheroids were treated with latent TGF $\beta 1$, the activation of the Smad3 pathway could also be inhibited if ITGB6 was blocked by a specific antibody. After the expression of SMYD3 or ITGB6 was altered, the expression of N-cadherin, Snail, Vimentin and E-cadherin and the morphological structure of the spheroids were consequently changed. Hence, we determined that ITGB6mediated TGF $\beta$ activation is involved in regulating the expression of N-cadherin, Snail, Vimentin and E-cadherin and promoting EMT progression in ovarian cancer spheroids. In addition, as the binding partner of ITGB6, ITGAV, had no independent effect on activating latent TGF $\beta 1$ in $3 \mathrm{D}$-cultured ovarian cancer spheroids. However, ITGAV might be play a role in the ITGB6-regulated activation of latent TGF $\beta 1$ in 3Dcultured ovarian cancer spheroids. Therefore, in future, more studies should be done to explore the role of ITGAV in TGF $\beta 1 /$ SMAD signaling activation. Interestingly, attenuating the expression of SMYD3 and ITGB6 also decreased the release of latent TGF $\beta 1$ from ovarian cancer spheroids, which further demonstrated the regulatory roles of SMYD3 and ITGB6 in TGF $\beta 1$ pathway activation.

Surprisingly, we also found that the expression of SMYD3 and ITGB6 could be upregulated after TGF $\beta 1$ treatment, which formed a positive feedback loop among SMYD3/ITGB6/TGF $\beta 1$ to enhance the invasion and adhesion of ovarian cancer spheroids by regulating the expression of E-cadherin, Ncadherin, Snail and Vimentin (Figure 7). Several previous studies also showed that TGF $\beta$ could induce the expression of SMYD3 and ITGB6. Denise et al. stated that SMYD3 was specifically regulated by TGF $\beta$ in iTreg cells (36). Mingyan Xu et al. reported that TGF $\beta 1$ could induce ITGB6 transcription via JunB- and CBP-mediated histone hyperacetylation in oral squamous cell carcinoma (37). Chao Jing et al. reported that miR-17/20a could reduce the expression of ITGB6 by attenuating the activation of TGF $\beta$ and phosphorylation of SMAD2/3 in esophageal squamous cell carcinoma (38).

In conclusion, we detailed one essential pathway that contributed to the SMYD3- and ITGB6-mediated enhancement of invasion and adherence in ovarian cancer spheroids. Our study also emphasized the significance of choosing SMYD3 and ITGB6 as potential targets for the treatment of ovarian cancer transcoelomic metastasis.

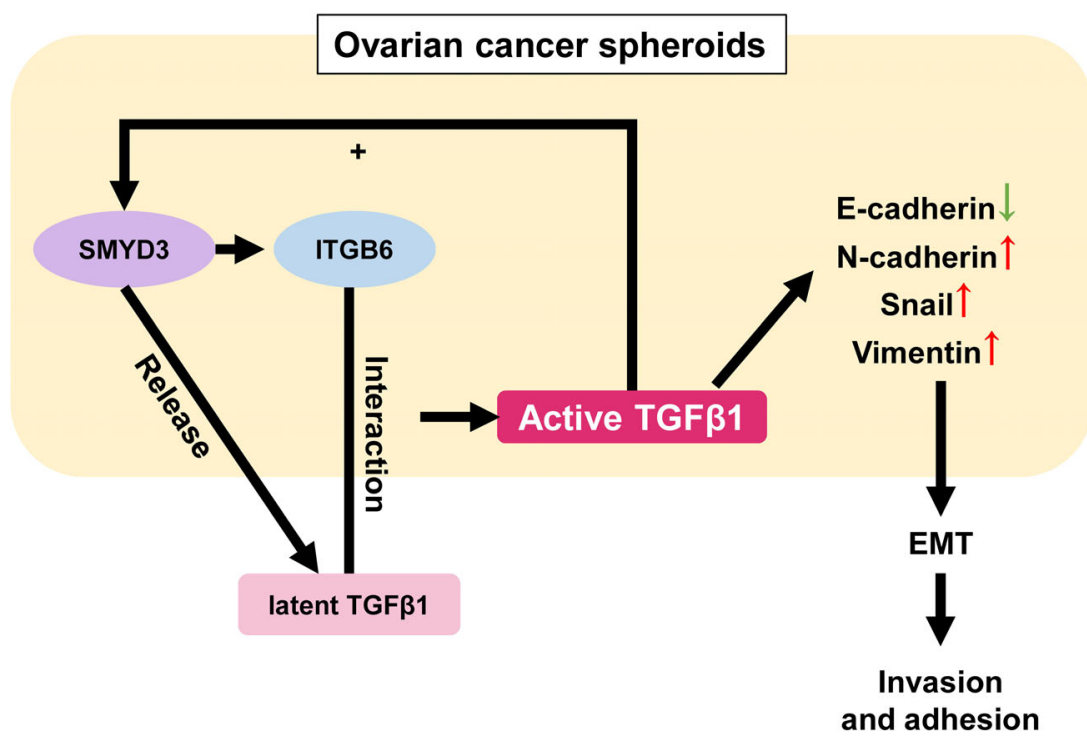

FIGURE 7 | Schematic model of the role of the SMYD3/ITGB6/TGFß1 positive feedback loop in promoting the invasion and adhesion of ovarian cancer spheroids. In ovarian cancer spheroids, SMYD3 can increase the expression of ITGB6. When latent TGF $\beta 1$ binds to ITGB6 on the cell surface, TGF $\beta 1$ is activated and upregulates the expression of $\mathrm{N}$-cadherin, Snail, Vimentin as well as downregulates E-cadherin to enhance the invasion and adhesion of ovarian cancer spheroids by stimulating the Smad3 pathway. Ovarian cancer spheroids can release more latent TGF $\beta 1$ at the same time. In addition, TGF $\beta 1$ can also facilitate the expression of SMYD3 and ITGB6 in a feedback manner. 


\section{DATA AVAILABILITY STATEMENT}

The raw data supporting the conclusions of this article will be made available by the authors, without undue reservation.

\section{ETHICS STATEMENT}

All experiments were approved by the Ethics Committee of Ruijin Hospital, Shanghai Jiao Tong University, School of Medicine. The informed consents were obtained from all patients included in the study.

\section{AUTHOR CONTRIBUTIONS}

All authors contributed to the article and approved the submitted version. $\mathrm{YJ}$ and $\mathrm{TZ}$ contributed in writing the manuscript, conducting the experiments, and doing the data analysis. YS helped to conduct part of the experiments. WF and TL designed this study and were the corresponding authors of this manuscript.

\section{REFERENCES}

1. Webb PM, Jordan SJ. Epidemiology of Epithelial Ovarian Cancer. Best Pract Res Clin Obstet Gynaecol (2016) 41:3-14. doi: 10.1016/j.bpobgyn.2016.08.006

2. Bray F, Ferlay J, Soerjomataram I, Siegel RL, Torre LA, Jemal A. Global Cancer Statistics 2018: GLOBOCAN Estimates of Incidence and Mortality Worldwide for 36 Cancers in 185 Countries. CA: Cancer J Clin (2018) 68 (6):394-424. doi: 10.3322/caac.21492

3. Naora H, Montell DJ. Ovarian Cancer Metastasis: Integrating Insights From Disparate Model Organisms. Nat Rev Cancer (2005) 5(5):355-66. doi: $10.1038 / \mathrm{nrc1611}$

4. Shield K, Ackland ML, Ahmed N, Rice GE. Multicellular Spheroids in Ovarian Cancer Metastases: Biology and Pathology. Gynecol Oncol (2009) 113(1):143-8. doi: 10.1016/j.ygyno.2008.11.032

5. Jiang Y, Lyu T, Che X, Jia N, Li Q, Feng W. Overexpression of SMYD3 in Ovarian Cancer is Associated With Ovarian Cancer Proliferation and Apoptosis via Methylating H3K4 and H4K20. J Cancer (2019) 10(17):407284. doi: $10.7150 /$ jca. 29861

6. Bottino C, Peserico A, Simone C, Caretti G. SMYD3: An Oncogenic Driver Targeting Epigenetic Regulation and Signaling Pathways. Cancers (Basel) (2020) 12(1):142. doi: 10.3390/cancers12010142

7. Lyu T, Jiang Y, Jia N, Che X, Li Q, Yu Y, et al. SMYD3 Promotes Implant Metastasis of Ovarian Cancer via H3K4 Trimethylation of Integrin Promoters. Int J Cancer (2020) 146(6):1553-67. doi: 10.1002/ijc.32673

8. Massague J. TGFbeta in Cancer. Cell (2008) 134(2):215-30. doi: 10.1016/ j.cell.2008.07.001

9. Heldin CH, Landstrom M, Moustakas A. Mechanism of TGF-Beta Signaling to Growth Arrest, Apoptosis, and Epithelial-Mesenchymal Transition. Curr Opin Cell Biol (2009) 21(2):166-76. doi: 10.1016/j.ceb.2009.01.021

10. Wu M, Chen X, Lou J, Zhang S, Zhang X, Huang L, et al. TGF-Betal Contributes to CD8+ Treg Induction Through P38 MAPK Signaling in Ovarian Cancer Microenvironment. Oncotarget (2016) 7(28):44534-44. doi: 10.18632/oncotarget.10003

11. Bierie B, Moses HL. Transforming Growth Factor Beta (TGF-Beta) and Inflammation in Cancer. Cytokine Growth Factor Rev (2010) 21(1):49-59. doi: 10.1016/j.cytogfr.2009.11.008

12. Ahamed J, Burg N, Yoshinaga K, Janczak CA, Rifkin DB, Coller BS. In Vitro and In Vivo Evidence for Shear-Induced Activation of Latent Transforming Growth Factor-Beta1. Blood (2008) 112(9):3650-60. doi: 10.1182/blood-200804-151753

13. Annes JP, Munger JS, Rifkin DB. Making Sense of Latent TGFbeta Activation. J Cell Sci (2003) 116(Pt 2):217-24. doi: 10.1242/jcs.00229

\section{FUNDING}

This work was supported by the National Natural Science Foundation of China (grant number: 8217100345), Ruijin Youth NSFC Cultivation Fund (Grant No. 2019QNPY02014) and Guangci Distinguished Young Scholars Training Program of Shanghai Jiaotong University School of Medicine affiliated Ruijin Hospital (GCQN-2019-B12).

\section{ACKNOWLEDGMENTS}

We appreciated to all the efforts that was made by the surgeons in our department. And we would like to express our gratitude to the support given by National Natural Science Foundation of China (grant number: 8217100345), Ruijin Youth NSFC Cultivation Fund (Grant No. 2019QNPY02014) and Guangci Distinguished Young Scholars Training Program of Shanghai Jiaotong University School of Medicine affiliated Ruijin Hospital (GCQN-2019-B12).

14. Munger JS, Huang X, Kawakatsu H, Griffiths MJ, Dalton SL, Wu J, et al. The Integrin Alpha V Beta 6 Binds and Activates Latent TGF Beta 1: A Mechanism for Regulating Pulmonary Inflammation and Fibrosis. Cell (1999) 96(3):31928. doi: 10.1016/S0092-8674(00)80545-0

15. Vinci M, Box C, Eccles SA. Three-Dimensional (3D) Tumor Spheroid Invasion Assay. J Vis Exp: JoVE (2015) 99):e52686. doi: 10.3791/52686

16. ten Dijke P, Hill CS. New Insights Into TGF-Beta-Smad Signalling. Trends Biochem Sci (2004) 29(5):265-73. doi: 10.1016/j.tibs.2004.03.008

17. Shi Y, Zhang J, Liu M, Huang Y, Yin L. SMAD3 Inducing the Transcription of STYK1 to Promote the EMT Process and Improve the Tolerance of Ovarian Carcinoma Cells to Paclitaxel. J Cell Biochem (2019) 120(6):10796-811. doi: $10.1002 /$ jcb. 28371

18. Nilchian A, Giotopoulou N, Sun W, Fuxe J. Different Regulation of Glut1 Expression and Glucose Uptake During the Induction and Chronic Stages of Tgf $\beta 1$-Induced EMT in Breast Cancer Cells. Biomolecules (2020) 10(12):1621. doi: 10.3390/biom10121621

19. Ingthorsson S, Andersen K, Hilmarsdottir B, Maelandsmo GM, Magnusson MK, Gudjonsson T. HER2 Induced EMT and Tumorigenicity in Breast Epithelial Progenitor Cells is Inhibited by Coexpression of EGFR. Oncogene (2016) 35(32):4244-55. doi: 10.1038/onc.2015.489

20. Worthington JJ, Klementowicz JE, Travis MA. Tgf $\beta$ : A Sleeping Giant Awoken by Integrins. Trends Biochem Sci (2011) 36(1):47-54. doi: 10.1016/ j.tibs.2010.08.002

21. Hawinkels LJ, Verspaget HW, van der Reijden JJ, van der Zon JM, Verheijen JH, Hommes DW, et al. Active TGF-Beta1 Correlates With Myofibroblasts and Malignancy in the Colorectal Adenoma-Carcinoma Sequence. Cancer Sci (2009) 100(4):663-70. doi: 10.1111/j.1349-7006.2009.01100.x

22. Duperret EK, Dahal A, Ridky TW. Focal-Adhesion-Independent Integrin- $\alpha v$ Regulation of FAK and C-Myc Is Necessary for 3D Skin Formation and Tumor Invasion. J Cell Sci (2015) 128(21):3997-4013. doi: $10.1242 /$ jcs. 175539

23. Khan Z, Marshall JF. The Role of Integrins in Tgf $\beta$ Activation in the Tumour Stroma. Cell Tissue Res (2016) 365(3):657-73. doi: 10.1007/s00441-016-2474-y

24. Webb PM, Jordan SJ. Epidemiology of Epithelial Ovarian Cancer. Best Pract Res Clin Obstet Gynaecol (2017) 41:3-14. doi: 10.1016/j.bpobgyn.2016.08.006

25. Yeung TL, Leung CS, Yip KP, Au Yeung CL, Wong ST, Mok SC. Cellular and Molecular Processes in Ovarian Cancer Metastasis. A Review in the Theme: Cell and Molecular Processes in Cancer Metastasis. Am J Physiol Cell Physiol (2015) 309(7):C444-56. doi: 10.1152/ajpcell.00188.2015

26. Matei D, Nephew KP. Epigenetic Attire in Ovarian Cancer: The Emperor's New Clothes. Cancer Res (2020) 80(18):3775-85. doi: 10.1158/00085472.CAN-19-3837 
27. Cancer Genome Atlas Research Network. Integrated Genomic Analyses of Ovarian Carcinoma. Nature (2011) 474(7353):609-15. doi: 10.1038/ nature10166

28. Zhu T, Chen R, Wang J, Yue H, Lu X, Li J. The Prognostic Value of ITGA and ITGB Superfamily Members in Patients With High Grade Serous Ovarian Cancer. Cancer Cell Int (2020) 20:257. doi: 10.1186/s12935-020-01344-2

29. Bengs S, Becker E, Busenhart P, Spalinger MR, Raselli T, Kasper S, et al. $\beta(6)$ -Integrin Serves as a Novel Serum Tumor Marker for Colorectal Carcinoma. Int J Cancer (2019) 145(3):678-85. doi: 10.1002/ijc.32137

30. Cao D, Qi Z, Pang Y, Li H, Xie H, Wu J, et al. Retinoic Acid-Related Orphan Receptor C Regulates Proliferation, Glycolysis, and Chemoresistance via the PD-L1/ITGB6/STAT3 Signaling Axis in Bladder Cancer. Cancer Res (2019) 79 (10):2604-18. doi: 10.1158/0008-5472.CAN-18-3842

31. Xu M, Yin H, Cai Y, Huang W, Ji Q, Liu F, et al. Lysophosphatidic Acid Induces Integrin $\beta 6$ Expression in Human Oral Squamous Cell Carcinomas Cells via LPAR1 Coupling to $\mathrm{G} \alpha(\mathrm{I})$ and Downstream SMAD3 and ETS-1 Activation. Cell Signal (2019) 60:81-90. doi: 10.1016/ j.cellsig.2019.04.008

32. Ganesh K, Massagué J. Targeting Metastatic Cancer Cancer Genome Atlas Research Network. Nat Med (2021) 27(1):34-44. doi: 10.1038/s41591-02001195-4

33. Papageorgis P. Tgf $\beta$ Signaling in Tumor Initiation, Epithelial-ToMesenchymal Transition, and Metastasis. J Oncol (2015) 2015:587193. doi: $10.1155 / 2015 / 587193$

34. Puthawala K, Hadjiangelis N, Jacoby SC, Bayongan E, Zhao Z, Yang Z, et al. Inhibition of Integrin Alpha(V)Beta6, an Activator of Latent Transforming Growth Factor-Beta, Prevents Radiation-Induced Lung Fibrosis. Am J Respir Crit Care Med (2008) 177(1):82-90. doi: 10.1164/rccm.200706-806OC

35. Koth LL, Alex B, Hawgood S, Nead MA, Sheppard D, Erle DJ, et al. Integrin Beta6 Mediates Phospholipid and Collectin Homeostasis by Activation of
Latent TGF-Beta1. Am J Respir Cell Mol Biol (2007) 37(6):651-9. doi: 10.1165/ rcmb.2006-0428OC

36. Nagata DE, Ting HA, Cavassani KA, Schaller MA, Mukherjee S, Ptaschinski C, et al. Epigenetic Control of Foxp3 by SMYD3 H3K4 Histone Methyltransferase Controls Itreg Development and Regulates Pathogenic TCell Responses During Pulmonary Viral Infection. Mucosal Immunol (2015) 8 (5):1131-43. doi: 10.1038/mi.2015.4

37. Xu M, Yin L, Cai Y, Hu Q, Huang J, Ji Q, et al. Epigenetic Regulation of Integrin $\beta 6$ Transcription Induced by TGF- $\beta 1$ in Human Oral Squamous Cell Carcinoma Cells. J Cell Biochem (2018) 119(5):4193-204. doi: 10.1002/jcb.26642

38. Jing C, Ma G, Li X, Wu X, Huang F, Liu K, et al. MicroRNA-17/20a Impedes Migration and Invasion via TGF- $\beta /$ ITGB6 Pathway in Esophageal Squamous Cell Carcinoma. Am J Cancer Res (2016) 6(7):1549-62.

Conflict of Interest: The authors declare that the research was conducted in the absence of any commercial or financial relationships that could be construed as a potential conflict of interest.

Publisher's Note: All claims expressed in this article are solely those of the authors and do not necessarily represent those of their affiliated organizations, or those of the publisher, the editors and the reviewers. Any product that may be evaluated in this article, or claim that may be made by its manufacturer, is not guaranteed or endorsed by the publisher.

Copyright (c) 2021 Jiang, Zhou, Shi, Feng and Lyu. This is an open-access article distributed under the terms of the Creative Commons Attribution License (CC BY). The use, distribution or reproduction in other forums is permitted, provided the original author(s) and the copyright owner(s) are credited and that the original publication in this journal is cited, in accordance with accepted academic practice. No use, distribution or reproduction is permitted which does not comply with these terms. 